\title{
JOGOS DE REGRAS E EXPERIÊNCIA PEDAGÓGICA COM IDOSOS: REVISÃO DA PRODUÇÃO ACADÊMICA BRASILEIRA 2007- $2017^{1}$
}

\author{
Mariana Nascimento ${ }^{2}$ \\ Geiva Calsa ${ }^{3}$
}

\begin{abstract}
Resumo: Objetivou-se investigar na produção científica brasileira estudos sobre idosos, jogos de regras e teoria piagetiana nos últimos dez anos - 2007 a 2017. A partir do estudo de revisão, foram encontradas apenas três pesquisas sobre o tema. Os trabalhos selecionados ressaltam a importância dos jogos de regras para o desenvolvimento intelectual e afetivo em idosos. As sessões pedagógicas com os jogos de regras, adaptadas do método clínico, permitiram aos participantes aprimorar suas estratégias e consequentemente melhorar seu desempenho no jogo.

Palavras-chave: Educação. Idoso. Jogos de regras. Construtivismo
\end{abstract}

\section{Game rules and educational experience with elderly: academic a look on the Brazilian academic production 2007-2017}

\begin{abstract}
It was aimed to investigate the Brazilian scientific production studies on the elderly, rules of games and Piaget's theory in the past decade - from 2007 to 2017. From the review of the literature, we found only three studies on the subject. The selected works emphasize the importance of the games rules for intellectual and emotional development in the elderly. The teaching sessions with game rules, adapted from the clinical method, allowed participants to improve their strategies and thus improve their performance in the game.
\end{abstract}

Keywords: Education. Elderly. Game Rules. Constructivism.

\footnotetext{
' O presente artigo é um recorte de nossa dissertação de mestrado intitulada A tomada de consciência como possibilidade de proteção de idosos da UNATI: uma experiência pedagógica com o jogo Quarto, defendida em 15 de fevereiro de 2016, por meio do Programa de Pós-Graduação em Educação da Universidade Estadual de Maringá/PR (UEM) e sob a orientação da Profa. Dra. Geiva Carolina Calsa. Parte deste trabalho foi enviado para os Anais da XXI Semana de Pedagogia, X Encontro de Pesquisa em Educação (UEM).

${ }^{2}$ Universidade Estadual de Maringá. (marianacostadonascimento@gmail.com)

${ }^{3}$ Universidade Estadual de Maringá (gccalsa@uem.br)
}

$$
\text { v. } 21 \text { n. } 45 \text { p. } 28(-293 \quad \text { jan/mar. } 2019 \text {. }
$$




\section{INTRODUÇÃO}

Nos últimos anos, observamos um crescente número de pesquisadores debruçarem-se sobre o tema do envelhecimento, com ampliação da produção científica, principalmente nas áreas da saúde e da psicologia. Isso porque a velhice e problemas sociais decorrentes tem-se tornado um tema emergente em nosso país e mundialmente. Todavia, como nos alerta Barros (2006) a transformação da velhice em um problema social não é resultado apenas do aumento demográfico de idosos, mas também ao reconhecimento público deste grupo, assim como sua legitimação social (a partir da criação de leis, como o Estatuto do Idoso, direito a aposentadoria), construção de uma especialidade científica nesta área - a Gerontologia. Tais aspectos vêm fornecendo novos significados para a velhice e o processo de envelhecimento, em particular desde a segunda metade do século XX.

Desse modo, os avanços nas pesquisas sobre o envelhecimento, bem como a aprovação das legislações, entre outras, vêm contribuindo para melhoria progressiva da qualidade de vida dos idosos. Aos poucos, foi sendo revisto o conceito clássico de envelhecimento como um processo negativo. "A velhice passou a ser vista também como um momento da vida na qual se pode viver com prazer, satisfação, realização pessoal, de maneira mais madura e também produtiva" (CACHIONI, FALCÃO, 2011, p. 179-180). Essa também é a posição de Park e Groppo (2009), para quem o aumento da expectativa de vida e as transformações legais para essa faixa etária possibilitaram um novo sentido para a velhice. Dessa maneira, chegar à velhice deixou de significar simplesmente a submissão a doenças ou à morte.

As novas representações sobre velhice trouxeram mudanças não apenas nos âmbitos sociais e legais, mas também para a área educacional. Nesta área, observamos a criação dos primeiros grupos de convivência de idosos por meio de inciativas do SESC (Serviço Social do Comércio), na década de 1960 e das Universidades Abertas a partir de 1980. De acordo com Cachioni (1998, p. 3), "além de proporcionar vantagens para a população idosa, essas iniciativas têm proporcionado espaços e oportunidades para a discussão do fenômeno velhice e, também, para a investigação de suas características no país”.

As escolas e Universidades Abertas à Terceira Idade ofertam palestras e debates sobre temas cotidianos, velhice e processo de envelhecimento, além de promoverem oficinas de música, arte, dança, teatro, ginástica, artesanato, entre 
outras atividades (TEIXEIRA, 2008; CACHIONI, FALCÃO, 2011; TAAM, STIELTJES, 2011). Nesse sentido, com base na proposta pedagógica destes centros, verificamos, a partir da literatura acadêmica, as possibilidades dos jogos de regras se constituírem instrumentos didáticos em atividades com idosos, como as que ocorrem nas Universidades Aberta à Terceira Idade.

Partindo dos pressupostos da teoria piagetiana, constatamos em estudos recentes que os jogos de regras podem se constituir ferramentas relevantes para o trabalho pedagógico com idosos, uma vez que favorece o desenvolvimento como propaga esta vertente, segundo a qual a inteligência não é "estática, prédeterminada [...], pelo contrário, a inteligência se mantém em um constante movimento de evolução e construção de novos conhecimentos” (SANTOS, 2007 , p. 21). Para a autora "os aspectos cognitivos são fundamentais para um desenvolvimento pleno, e precisam fazer parte dos programas de saúde pública e dos objetivos dos centros de convivência para a terceira idade" (p. 117).

Para Camargo (2015) o jogo faz parte da atividade humana desde a infância. Na criança o jogo refere-se a uma atividade rotineira que possuem horários previstos pela família e pela escola. E embora o olhar do adulto esteja mais adaptado a observar o jogo infantil e não o seu próprio jogar, esta atividade está presente em boa parte de suas atividades cotidianas. Entre essas atividades a autora destaca as "sérias e que são tratadas, também, como diversão, porque são prazerosas para o sujeito" ou quando "o sujeito as realiza pelo prazer de realizar e não apenas porque precisam ser feitas” (p. 142-143).

A autora explica que, embora o adulto já tenha vivenciado todos os estádios de desenvolvimento do jogo conforme descrito pela teoria piagetiana (exercício, simbólico e de regras), continua exercitando-os ainda que não se dê conta disso. O jogo de exercício está presente em várias situações que o adulto vive no dia a dia como "na aprendizagem de uma dança, por exemplo", uma vez que os ensaios são preenchidos basicamente por imitações e repetições. "O jogo simbólico aparece nas fantasias, na dramaturgia”, bem como nos diferentes papéis e funções sociais que os adultos assumem no cotidiano pessoal e profissional. Seu desempenho depende de posturas e linguagens diversas e adequadas a cada contexto. Os jogos de regras por sua vez são vividos nas atividades esportivas e profissionais que envolvem planejamento, execução e reorganização de estratégias (CAMARGO, 2015, p. 143).

Dell'Agli e Brenelli (2007), Rebeiro (2012) e Carvalho (2013) em estudos com crianças evidenciam a importância dos jogos de regras para o desenvolvimento de aspectos cognitivos, sociais e afetivos desses sujeitos. Como 
exemplo disso, Rebeiro (2012) identificou fatores cognitivos durante o processo de planejamento e criação de estratégias para vencer o jogo, afetivos quando enfrentam ou abandonam as dificuldades e tensões geradas pelas jogadas e os sociais em situações de empatia e trocas entre os jogadores.

Dipe e Gaio (2007, p.248) também reforçam as conclusões dessas pesquisas, assinalando que a atividade lúdica pode contribuir para o desenvolvimento do pensamento uma vez que nela "[...] está presente a ferramenta mais adequada para o processo de construção do conhecimento, o desequilíbrio, o qual permite a tomada de consciência, tornando possível a reflexão (pensar por si mesmo)”.

Encontramos em Macedo (1994, p.71) uma explicação para essa condição de desenvolvimento na medida em que os jogos favorecem a reflexão do sujeito sobre seus erros durante sua ação permitindo-os retomar e reorganizar o seu pensar e o seu fazer. Os jogos "criam um contexto que se pode mostrar" aos indivíduos ou eles podem verificar por si mesmos, "a contradição, o conflito e a não-coerência entre suas respostas” favorecendo a compreensão de suas ações.

De acordo com Frias (2009) a atividade lúdica constitui-se um desafio para o indivíduo e para suas relações com o outro, pois implica a vivência de sentimentos e emoções como medo, inveja, ódio, raiva, disputa, cooperação, entre outros. Além disso, como explica Macedo, Petty e Passos (2005, p.49), o jogo pode vir a tornar-se um desafio para o indivíduo pois leva-o a buscar respostas para problemas que surgem continuamente e, neste percurso, pode vitr. A construir novos saberes e fazeres. Diante de uma situação-problema, o indivíduo acaba por "[...] investigar, prestar mais atenção nas suas ações e nos resultados que elas produziram para obter êxito”. Em outras palavras, refletir, transformar e reorganizar o seu pensamento e suas ações.

Para as relações do indivíduo com o outro o jogo constitui-se um desafio também porque, entre outros aspectos, para ganhar é preciso "[...] desenvolver habilidades, estar atento, concentrado atender a outras exigências. Os jogos de regras são importantes por seu caráter competitivo, compreendendo-se competir como uma característica funcional, sem seu possível significado relacionado ao egoísmo”. Para Frias (2009, p. 30), a competição presente nos jogos de regras faz parte do desenvolvimento do sujeito que neste movimento busca superar e enfrentar desafios intelectuais, emocionais e sociais decorrentes de sua interação com o outro. 
A fecundidade do trabalho com os jogos de regras para o desenvolvimento dos indivíduos tem levado vários pesquisadores fundamentados na teoria piagetiana a realizar estudos privilegiadamente com crianças. Isso nos levou a perguntar sobre a existência de produção acadêmica sobre o tema entre a população idosa cuja volta ao processo de aprendizagem tem sido intensa, seja assumindo novos empregos, profissões ou voltando ao ambiente escolar. Em vista disso, neste artigo apresentamos os resultados de uma pesquisa bibliográfica sobre a produção acadêmica brasileira dos últimos dez anos envolvendo idosos e jogos de regras a partir do referencial piagetiano.

\section{MATERIAIS E MÉTODO}

Desenvolveu-se um estudo bibliográfico conforme as etapas sugeridas por Lara e Molina (2011): identificação, localização, compilação e fichamento. A primeira referiu-se à identificação das fontes a serem investigadas; na segunda localizaram-se e selecionaram-se os bancos de dados da web para obtenção das informações; na terceira reuniu-se e realizou-se a leitura flutuante do material encontrado; na quarta etapa, após leitura focada realizaram-se anotações e fichamento do material recolhido. A busca foi realizada ${ }^{4}$ no Banco de Teses e Dissertações Nacionais (BDTD), CAPES (Coordenação de Aperfeiçoamento de Pessoal de Nível Superior) e Scielo (Scientific Electronic Library Online) ${ }^{5}$ em língua portuguesa. Para isso, utilizamos combinações das palavras-chave e algumas de suas derivações: idoso (terceira idade, velho e velhice) jogo (jogo de regras) teoria piagetiana (Epistemologia Genética e construtivismo).

\section{RESULTADOS E DISCUSSÃO}

Nos últimos dez anos contemplados por esta revisão da produção acadêmica brasileira foram encontrados apenas três trabalhos que abordam ao mesmo tempo os temas idoso, jogo e teoria piagetiana: duas dissertações e uma tese (Quadro 1).

\footnotetext{
${ }^{4}$ A busca foi atualizada em 5 de abril de 2017.

"Os bancos BDTD, CAPES e Scielo foram selecionados por contemplar grande quantidade de estudos acadêmicos realizados em universidades de todo o território brasileiro.
} 


\begin{tabular}{|c|c|c|c|c|}
\hline Temas & Quantidade & Autor/a & $\begin{array}{c}\text { Tipo de } \\
\text { publicação }\end{array}$ & Título \\
\hline \multirow{3}{*}{$\begin{array}{c}\text { Idoso, } \\
\text { jogo e teoria } \\
\text { piagetiana }\end{array}$} & 3 & Santos (2007) & $\begin{array}{c}\text { Dissertaç } \\
\text { ào }\end{array}$ & $\begin{array}{l}\text { Análise microgenética de aspectos de } \\
\text { funcionamento de } \\
\text { adolescentes e de idosos por meio do } \\
\text { jogo Quoridor }\end{array}$ \\
\cline { 3 - 5 } & & Silva (2013) & $\begin{array}{c}\text { Dissertaç } \\
\text { ão }\end{array}$ & $\begin{array}{l}\text { Ojogo Set em adolescentes, adultos e } \\
\text { idosos: aspectos cognitivos }\end{array}$ \\
\cline { 3 - 5 } & Santos (2011) & Tese & $\begin{array}{l}\text { Análise microgenética de aspectos } \\
\text { cognitivos e afetivos em idosas: uma } \\
\text { proposta teórica e metodológica }\end{array}$ \\
\hline
\end{tabular}

Quadro 1 - Produção acadêmica envolvendo jogo, idoso e teoria piagetiana - 2007 a 2017

Fonte: Dados da pesquisa

O estudo de Claudimara Chisté Santos (2007) teve como objetivo investigar aspectos do funcionamento cognitivo em quatro adolescentes e quatro idosas por meio do jogo Quoridor. A pesquisa se desenvolveu em cinco etapas, sendo que as adolescentes e as idosas não tiveram encontros em conjunto, pois segundo a autora, a presença de outro grupo poderia alterar o comportamento e a concentração das participantes. No primeiro momento realizou uma entrevista com o intuito de obter informações sobre as participantes. Para complementar esses dados, foram utilizados dois testes psicométricos de memória e ajustamento emocional (TEMPLAN - Teste de Memória de Placas; EFN Escala Fatorial de Ajustamento). Durante a segunda etapa, as participantes conheceram o jogo Quoridor, a partir da manipulação das peças e entendimento das regras. A terceira etapa foi destinada as partidas em duplas. $\mathrm{O}$ número de partidas não foi delimitado previamente pela experimentadora, pois uma das jogadoras poderia sentir-se obrigadas a jogar, o que alteraria o resultado do estudo. Nesse sentido, houve negociação da seguinte regra: "a participante que sentisse cansada ou sentisse vontade de parar, falaria e as demais interromperiam o campeonato" (ibidem, p. 65). As idosas acabaram jogando um total de dez partidas, enquanto as adolescentes trinta partidas. Na quarta etapa, as jogadoras assistiram gravações de suas próprias jogadas, a fim de tentar identificar e explicar as intenções e objetivos de suas ações no jogo. Durante a quinta etapa foram propostas quatro situações-problema com o jogo Quoridor. As sessões de intervenção com o jogo revelaram níveis de tomada de consciência e de compreensão do jogo por parte dos idosos, pois o contato com o jogo permitiu as participantes construírem novos esquemas de ação e de 
pensamento. Os resultados encontrados pela pesquisadora reforçaram os de estudos anteriores realizados com adolescentes evidenciando a relevância deste jogo e de intervenção para as duas faixas etárias. Os dados sugerem ainda que a intervenção por meio do jogo de regras é válida "tanto para adolescentes que tenham necessidade de vivenciar um espaço educativo mais lúdico e construtivista quanto para idosas que necessitam se manter estimuladas" (ibidem, p. 110).

Em pesquisa posterior, Santos (2011) desenvolveu um conjunto de intervenções pedagógicas construtivistas com seis mulheres com idade entre 60 e 67 anos. O estudo apresentou como intuito investigar a relação entre cognição e afetividade durante partidas com o Jogo Cara a Cara. Para cumprir os objetivos, a pesquisa foi desenvolvida em sete etapas. Na primeira etapa a pesquisadora realizou-se uma entrevista semiestruturada individualmente, a fim de obter dados gerais dos participantes. Durante o segundo momento aplicou a entrevista clínica, também individual, cujo objetivo foi caracterizar a conduta de cada sujeito em relação aos aspectos afetivos (interesse, autovalorização e vontade). Na terceira etapa utilizou o instrumento de memória adaptado de Inhelder e Caprona (1979). Posteriormente, no quarto momento, as jogadoras conheceram o jogo Cara a Cara e iniciaram as partidas em duplas. Durante a quinta etapa foram propostas seis situações-problema com o jogo Cara a Cara. No sexto momento, com o intuito de caracterizar o tipo de conduta em relação aos aspectos afetivos (interesse, autovalorização e vontade) foram propostas três Histórias sobre os Aspectos Afetivos, adaptado do método clínico piagetiano. Nesta etapa foi realizada novamente a entrevista clínica de aspectos afetivos.

Os resultados apresentados pelas idosas indicaram forte relação entre cognição e afetividade em seu desempenho. A autora constatou que aspectos da afetividade das idosas participantes da pesquisa interferiram positivamente para o planejamento e organização de estratégias de jogo. Os resultados da pesquisa levaram a pesquisadora a concluir que "os aspectos cognitivos estruturam o desenvolvimento, mas a participação dos aspectos afetivos contribui para a manutenção ou afastamento da ação que pode desencadear o desenvolvimento" intelectual dos idosos (ibidem, p. 204-205). Suas conclusões de que à cognição "[...] cabe a tarefa de organizar e estruturar os esquemas e à afetividade cabe acelerar ou retardar a construção do conhecimento" (ibidem, p. 205) reforçam as conclusões de estudos anteriores de Piaget e colaboradores.

A dissertação de Sandreilane Cano da Silva (2013) apresentou como objetivo analisar aspectos cognitivos de adolescentes, adultos e idosos por meio 
do jogo Set. A pesquisa foi desenvolvida durante quinze encontros, com duração média de 90 minutos. Participaram da pesquisa 40 participantes (18 adolescentes, 12 adultos e 10 idosos) integrantes do Programa Escola da Família: desenvolvimento de uma cultura de paz no Estado de São Paulo. De acordo com a autora, o projeto apresenta como objetivo ofertar atividades relacionadas a área da cultura, esporte, saúde e qualificação profissional para a comunidade. Para a pesquisa, foram selecionados adolescentes estudantes do programa e adultos e idosos da comunidade local e/ou funcionários professores da instituição. No primeiro momento da pesquisa, os participantes conhecerem o jogo Set, manipularam as peças e aprenderam as regras. E no segundo momento foram propostas as partidas em dupla. Como a pesquisa objetivou comparar o desenvolvimento de adolescentes, jovens e idosos não houve mistura dos grupos etários durante as jogadas. Verificou-se a partir da proposta da oficina diferentes modos de jogar segundo a faixa etária. Entretanto, em todos os participantes foi evidenciado que a intervenção possibilitou o aperfeiçoamento das estratégias utilizadas.

Ao analisar os três estudos encontrados constatamos que dois (SANTOS, 2007; SILVA, 2013) deles investigaram os aspectos cognitivos envolvidos nas atividades de jogo de idosos e jovens buscando estabelecer similaridades entre ambos, enquanto um (SANTOS, 2011) pesquisou a presença da afetividade além dos elementos cognitivos na atividade lúdica do jogo com idosas. As conclusões de Santos (2011) reiteram as discussões da teoria piagetiana sobre a indissociação da cognição e da afetividade serem indissociáveis. Para Piaget (2014) toda a ação possui um elemento enérgico (afetivo) nas atividades dos indivíduos, embora apresentem dinâmicas diferentes. Conforme esses estudos a afetividade define as metas para as ações, enquanto a inteligência organiza os meios para que essas metas sejam desenvolvidas. $\mathrm{O}$ afeto desempenha papel essencial para o funcionamento da inteligência, pois sem este não haveria interesse e motivação para a formulação e resolução de problemas. Nenhum dos trabalhos analisou as possíveis correspondências e diferenças entre a forma de pensamento de jovens e idosos nas atividades de jogo realizadas nas pesquisas.

Em comum os trabalhos três estudos desenvolveram atividades de intervenção pedagógica por parte das pesquisadoras por meio de oficina de jogos de regras. Nestas oficinas as intervenções pedagógicas foram adaptadas do método clínico piagetiano. As pesquisas são diferentes no que tange às metodologias utilizadas para a coleta de informações e de análise dos dados. A pesquisa de Santos (2007) utilizou de dois testes psicométricos de memória e 
ajustamento emocional (TEMPLAN - Teste de Memória de Placas; EFN Escala Fatorial de Ajustamento) e de sessões de intervenção pedagógica com o jogo Quoridor. Em sua tese, Santos (2011) realizou entrevista clínica antes e pós-intervenção das sessões com o jogo Cara a Cara. E na pesquisa de Silva (2013) foram feitas sessões pedagógicas com o jogo Set. Quanto à análise das informações obtidas em seu estudo Santos (2007) estabelece níveis de compreensão do jogo e de TC, enquanto em sua segunda pesquisa Santos (2011) sistematizou níveis relativos aos aspectos afetivos e cognitivos presentes na atividade de jogo; por último, na pesquisa de Silva (2013) a autora analisou os aspectos cognitivos no jogo Set, mas não chegou a criar níveis.

Além de partidas e resolução de situações-problema no jogo como na pesquisa de Silva (2013) as duas pesquisas de Santos $(2007$; 2011) envolveram outras técnicas de pesquisa. Em sua dissertação de mestrado usou dois testes psicométricos, um teste de memória (TEMPLAN - Teste de Memória de Placas) e outro de ajustamento emocional à atividade (EFN - Escala Fatorial de Ajustamento). Em sua tese de doutorado, a autora valeu-se de outros dois tipos de técnicas, Entrevista Clínica e História dos aspectos afetivos, que, segundo a autora, lhe possibilitaram a avaliação da afetividade das idosas envolvida nos jogos. Apesar das diferenças nas pesquisas apresentadas seus resultados indicam convergência quanto à importância dos jogos de regras para o desenvolvimento intelectual e afetivo de idosos e jovens confirmando resultados anteriores em estudos realizados com outras faixas etárias (GARCIA, 2010; CAIADO, 2012; REBEIRO, 2012; QUINELATO, 2014).

Na pesquisa de Santos (2007), a maioria das participantes manifestaram níveis mais complexos de compreensão de jogo e de tomada de consciência. Em relação ao nível de compreensão do jogo, uma jovem e uma idosa iniciou em I e apresentou nível II/B; duas adolescentes e uma idosa iniciaram em I e manifestaram ao longo das partidas condutas de nível II/A; uma idosa e uma jovem iniciaram em II/A e manifestaram condutas de nível II/B e apenas uma idosa permaneceu em nível I ao longo das sessões de intervenção. Sobre os níveis de tomada de consciência, três jovens e uma idosa iniciaram em I e apresentaram condutas de nível II/B; uma adolescente e uma idosa iniciaram em nível I e manifestaram condutas de nível II/A e duas idosas começaram em I e manifestaram ações de nível II/C ao longo das sessões com o jogo Quoridor.

No trabalho de doutorado de Santos (2011), também é possível evidenciar que a maioria das participantes manifestou níveis mais complexos em relação aos aspectos afetivos e cognitivos. Os aspectos afetivos foram divididos em 
autovalorização, interesse e força de vontade. Sobre a autovalorização, quatro idosas manifestaram condutas de nível gama e duas de nível beta. Em relação ao interesse, três participantes apresentaram ações de nível gama; duas de nível beta e uma participante apresentou ações de nível alfa. E sobre o aspecto força de vontade, quatro idosas apresentaram ações de nível gama e duas manifestaram condutas de nível beta. Os aspectos cognitivos foram subdivididos em aspectos cognitivos de conduta (alfa, gama e beta), Heurística I e Memória I. Sobre os fatores cognitivos de conduta, três participantes manifestaram condutas de nível beta, uma idosa manifestou condutas de nível alfa e em duas participantes não foi possível perceber perturbação do sistema. E relação a heurística I, uma participante demonstrou características de nível $\mathrm{II} / \mathrm{B}$, quatro de nível II/A e apenas uma de nível I. Sobre os níveis de memória, quatro idosas manifestaram condutas de nível IV/B, uma participante apresentou nível IV/A e outra idosa manifestou ações de nível III.

Embora a pesquisa de Silva (2013) não tenha estabelecido níveis de compreensão do jogo ou de tomada de consciência, os resultados da pesquisa indicaram que as sessões pedagógicas com o jogo Set permitiram aos participantes aprimorar suas estratégias e consequentemente melhorar seu desempenho no jogo.

Resultados próximos aos encontrados nas três pesquisas com referência aos idosos são reforçados pelos resultados de nossa pesquisa de mestrado (NASCIMENTO, 2016). O estudo realizado com doze estudantes de uma Universidade Aberta à Terceira Idade (UNATI-UEM/PR) revelou que sessões de intervenção pedagógica com uso do jogo Quarto favoreceram o desenvolvimento intelectual dos idosos. As verbalizações solicitadas pela experimentadora possibilitaram aos idosos-participantes aprimorar suas estratégias e consequentemente melhorar seu desempenho no jogo Quarto com maior quantidade de vitórias. Dessa forma disponível no sistema cognitivo dos idosos, conclui-se que a tomada de consciência pode vir a constituir um processo a ser utilizado em situações-problema do cotidiano. Entendemos que a meta reflexão possibilita aos sujeitos maior poder de decisão sobre seus problemas, mostrando-se mais autônomos e menos compassivos diante das dificuldades do meio. 


\section{CONSIDERAÇÕES FINAIS}

Os trabalhos encontrados durante a revisão da produção acadêmica brasileira da última década evidenciam a existência de poucos estudos sobre o uso de jogo de regras com idosos. Verificamos também que a quase totalidade das pesquisas sobre jogo tem sido realizadas com crianças ou jovens, mesmo as que são referenciadas na teoria piagetiana.

Em sua revisão da produção acadêmica, Santos (2007) já indicava que a quantidade de pesquisas utilizando jogos de regras dobrou desde o início dos anos 2000, sendo 86,5\% desses baseadas no referencial teórico piagetiano. Todavia, deste total apenas $2 \%$ tem envolvido trabalhos com idosos.

Essa constatação revela lacunas na produção acadêmica sobre o tema tanto do ponto de vista teórico como aplicado. Do ponto de vista teórico, o fato da Epistemologia Genética compreender a inteligência em constante desenvolvimento e transformação leva-nos a tentar compreender como se dá esta construção em todas as faixas etárias, incluindo os idosos cujo contingente vem aumentando vertiginosamente em nosso país. Do ponto de vista aplicado, em particular o educacional, a ampliação desses estudos permite aos educadores uma capacitação mais adequada para o atendimento de idosos em ambiente escolar, uma vez que poderiam utilizar-se dos jogos de regras para o seu desenvolvimento cognitivo e afetivo.

O presente artigo faz um convite aos pesquisadores desta área a aprofundarem as investigações sobre as possibilidades de desenvolvimento dos idosos por meio de jogos de regras, entre outros, a partir de pesquisas empíricas e discussões teóricas.

\section{REFERÊNCIAS}

BARROS, Myriam Moraes Lins de. In: (Org.). Velhice ou terceira idade? Rio de Janeiro: Editora FGV, 2006.

CACHIONI, Meire. Envelhecimento bem-sucedido e participação numa Universidade para a terceira idade. 1998. 122 f. Dissertação (Mestrado) - 
Programa de Pós-graduação em Educação, Universidade Estadual de Campinas, Campinas, 1998.

CACHIONI, Meire.; FALCÃO, Deusivania Vieira Silva. Velhice e educação: possibilidades e benefícios para a qualidade de vida. In: FALCÃO, D.V..S.; ARAÚJO, L.F. (Orgs.) Psicologia do envelhecimento: relações sociais, bem estar subjetivo e atuação profissional em contextos diferenciados. Campinas, Alínea, 2011, p. 175-194.

CAIADO, Ana Paulo Sthel. A regra em jogo: um estudo sobre a prática dos jogos de regras e o desenvolvimento moral infantil. 2012. $124 \mathrm{f}$. Tese

(Doutorado) - Programa de Pós-Graduação em Psicologia, Universidade de São Paulo, São Paulo, 2012.

CARVALHO, Luciana Ramos Rodrigues de. Oficinas com o jogo Set na sala de apoio à aprendizagem como espaço para pensar a resiliência. 2013. $186 \mathrm{f}$. Dissertação (Mestrado) - Programa de Pós-Graduação em Educação, Universidade Estadual de Londrina, Londrina, 2013.

DELL'AGLI, Betânia Alves Veiga; BRENELLI, Rosely Palermo. O jogo descubra o animal: um recurso no diagnóstico psicopedagógico. Psicologia em Estudo, Maringá, v. 12, n.3, p. 563-572, set./dez. 2007.

DIPE, Vânia Cristina; GAIO, Roberta. Educação Física, a construção do brinquedo e a descentração do pensamento infantil. Movimento \& Percepção, Espírito Santo do Pinhal, v. 7, n.10, p. 246-262, jan./jun.2007.

FRIAS, Eduardo Ribeiro. Jogo das representações (RPG) e Aspectos da Moral Autônoma. 2009. 110 f. Dissertação (Mestrado) - Programa de Pós-Graduação em Psicologia, Instituto de Psicologia da Universidade de São Paulo, São Paulo, São Paulo, 2009.

GARCIA, H. H. G. O. Adolescentes em grupo: aprendendo a cooperar em oficinas de jogos. 2010. 276 f. Tese (Doutorado) - Programa de Pós-

Graduação em Psicologia, Instituto de Psicologia da Universidade de São Paulo, São Paulo, São Paulo, 2010.

MACEDO, Lino de. Ensaios construtivistas. São Paulo: Casa do Psicólogo, 1994.

MACEDO, Lino de; PETTY, Ana Lúcia Sícoli; PASSOS, Nortimar Christe. Os jogos e o lúdico na aprendizagem escolar. Porto Alegre: Artmed, 2005. 
NASCIMENTO, Mariana Costa do. A tomada de consciência como possibilidade de proteção dos idosos: uma experiência pedagógica com o jogo Quarto na UNATI/UEM. 166 f. 2016. Dissertação (Mestrado) - Programa de Pós-Graduação em Educação, Universidade Estadual de Maringá, Maringá, 2016.

PARK, Margareth Brandini; GROPPO, Luís Antonio. In: (Orgs.). Educação e velhice. Holambra: Editora Setembro, 2009.

PIAGET, Jean. A formação do símbolo na criança: imitação, jogo e sonho, imagem e representação. Rio de Janeiro: Zahar, 1978.

REBEIRO, Gisele Bueno de Farias. Fatores protetivos e o jogo de regras Rammikub: um estudo com alunos do $6^{\circ}$ ano do Ensino Fundamental. 2012. 119 f. Dissertação (Mestrado) - Programa de Pós-Graduação em Educação, Universidade Estadual de Londrina, Londrina, 2012.

QUINELATO, Patrícia Thomásio. Fazer e compreender no jogo Sudoku e em situações-problema: um estudo com alunos do $9^{\circ}$ ano do Ensino Fundamental. 2014. 221 f. Tese (Doutorado) - Programa de Pós-Graduação em Psicologia, Universidade de São Paulo, São Paulo, 2014.

SANTOS, Claudimara Chisté; Análise microgenética de aspecto do funcionamento cognitivo de adolescentes e de idosos por meio do jogo quoridor. 2007. 145 f. Dissertação (Mestrado) - Programa de Pós-graduação em Educação, Universidade Federal do Espirito Santo, Vitória, 2007.

Análise microgenética de aspectos cognitivos e afetivos em idosas: uma proposta teórica e metodológica. 2011. 228 f. Tese (Doutorado) Programa de Pós-graduação em Educação, Universidade Federal do Espirito Santo, Vitória, 2011.

SILVA, Sandreline Cano da. O jogo Set em adolescentes, adultos e idosos: aspectos cognitivos. 2013. 124 f. Dissertação (Mestrado) - Programa de Pósgraduação em Psicologia, Universidade de São Paulo, São Paulo, 2013.

TAAM, Regina; STIELTJES, Cláudio. A UNATI da UEM: educação e política. IN: OLIVEIRA, Rita de Cássia da Silva; D’ALENCAR, Raimunda (Orgs.). As experiências de universidades abertas em um Brasil que envelhece. Curitiba: CRV, 2011, p. 141-159. 
TEIXEIRA, Solange Maria. Envelhecimento e trabalho no tempo do capital: implicações para a proteção social no Brasil. São Paulo: Cortez, 2008.

Recebido em 21/04/2017 Aprovado em 27/08/2018 